\title{
Effect of Brownian Diffusion on Squeezing Elastico-Viscous Nanofluid Flow with Cattaneo-Christov Heat Flux Model in a Channel with Double Slip Effect
}

\author{
Md Enamul Karim1,2, Md Abdus Samad1 \\ ${ }^{1}$ Department of Applied Mathematics, University of Dhaka, Dhaka, Bangladesh \\ ${ }^{2}$ Department of Mathematics, Comilla University, Cumilla, Bangladesh \\ Email: ekarim_du@yahoo.com, ekarim777@gmail.com,samad@du.ac.bd
}

How to cite this paper: Karim, M.E. and Samad, M.A. (2020) Effect of Brownian Diffusion on Squeezing Elastico-Viscous Nanofluid Flow with Cattaneo-Christov Heat Flux Model in a Channel with Double Slip Effect. Applied Mathematics, 11, 277-291. https://doi.org/10.4236/am.2020.114021

Received: February 20, 2020

Accepted: April 6, 2020

Published: April 9, 2020

Copyright $\odot 2020$ by author(s) and Scientific Research Publishing Inc. This work is licensed under the Creative Commons Attribution International License (CC BY 4.0).

http://creativecommons.org/licenses/by/4.0/

\begin{abstract}
The present study deals with the analysis of heat transfer of the unsteady Maxwell nanofluid flow in a squeezed rotating channel of a porous extensile surface subject to the velocity and thermal slip effects incorporating the theory of heat flow intensity of Cattaneo-Christov model for the expression of the energy distribution in preference to the classical Fourier's law. A set of transformations is occupied to renovate the current model in a system of nonlinear ordinary differential equations that are numerically decoded with the help of MATLAB integrated function bvp4c. The effects of various flow control parameters are investigated for the momentum, temperature and diffusion profiles, as well as for the wall shearing stress and the heat and mass transfer. The results are finally described from the material point of view. A comparison of heat flux models of Cattaneo-Christov and Fourier is also performed. An important result from the present work is that the squeezing parameter is strong enough in the middle of the channel to retard the fluid flow.
\end{abstract}

\section{Keywords}

Maxwell Fluid Model, Squeezing Flow, Elastico-Viscous, Nanofluid, Channel, Rotation

\section{Introduction}

The heat transfer phenomenon is of great concern because of its impact on industrial applications, including cooling of space and nuclear reactors, heat con- 
duction in tissues pasteurization of milk, magnetic targeting of drugs, etc. Fourier [1] proposed a heat flux model, named as heat conduction law that produces a parabolic energy equation that advocates an instantaneous change in the temperature of considered system at the beginning of any process. Cattaneo [2] introduced thermal relaxation time so as to produce the hyperbolic energy equation which permitted the heat transport through the transmission of thermal waves with finite speed. The theory of Cattaneo was further improved by Christov [3] who replaced the time derivative in the Cattaneo's model by the Oldroyd's upper-convective derivative [4] that preserved the material-invariant formulation and that became prominent as Cattaneo-Christov heat flux. Ciarletta and Straughan [5] analyzed the stability and uniqueness of the solution of the energy equation for Cattaneo-Christov heat flux model. Thermal relaxation time can be interpreted physically as the time needed for accumulating the thermal energy essential for generating heat flux [6] [7]. The inclusion of the thermal inertial in heat prorogation has effects in the heat transport in nano-material, nanofluids and many areas of ballistics and astrophysics [8] [9] [10].

The practice of adding polymers to mineral oils, known as multi-grade oils, has become recognized since the middle of 1990s [11] [12] [13]. These additions force the resulting lubricants to become non-Newtonian and viscoelastic exerting shear-rate dependent viscosity [14] [15]. The highly non-linear relationship between shear stress and strain rate of non-Newtonian fluids cannot be demonstrated by the classical Newtonian fluid model enclosing Navier-Stokes equations [16] [17]. The non-Newtonian fluids include polymer solutions, certain lubricants and oils, suspension and colloidal solutions, blood, melts, condensed milk, emulsions, soaps, shampoos, tomato paste, and many others containing the properties of both elasticity and viscosity. Researchers have proposed several viscoelastic fluid models incorporating different features of non-Newtonian fluids [18] [19] [20]. Models of viscoelastic fluids, including second-order fluids and/or Walter-B fluids, are ideal for slow motion of low elastic fluids [21]. To carry out an effective theoretical work in the industrial sector, the most realistic and practical viscoelastic fluid models, such as the Maxwell fluid or the Oldroyd-B fluid, should be considerable for efficient analysis [22] [23]. The Maxwell fluid, a simple class of rate- type viscoelastic material, eliminates the complex effects of shear-reliant viscosity and accordingly permits one to highlight the influence of fluids elasticity on the characteristics of its boundary layer [24]. Harris [25] first developed the constitutive equation of upper convected Maxwell (UCM) fluid to model the lubricant behavior of the non-Newtonian fluid. Due to the rising practical applications in industrial and manufacturing procedures, researchers have paid their attention to the study of boundary layer flow of non-Newtonian fluids [26] [27].

Nanofluids are the new-generation heat transfer fluids that contain higher thermal conductivity at very low particle concentrations than the conventional fluids. This idea of nanofluid was first developed by Choi [28]. Recent research- 
ers have identified that the substitution of usual coolants with nanofluids may be advantageous in processes like improving heat transfer efficiency in nuclear space and engineering, domestic refrigerators/freezers; and cooling of engine and micro-electronics [29]. Moreover, electromagnetic nanoparticles are playing important role in bio-medicinal applications as compared to other metallic particles, because these can be used to control and manipulate the nanofluid through magnetic force [30] [31]. As a part of these researches, Buongiorno [32] composed a mathematical model to study the convective heat transfer in nanofluids taking two important effects, namely the Brownian and thermophoresis diffusions into account.

While Stefan [33] carried out this pioneering work and basic formulations on flow phenomena, so far the analysis of the compression flow process is receiving considerable attention by the researchers because of its purposes in the fields of biomechanics and chemical engineering [34]. Reciprocating engine bearing performance, injection and compression molding, polymer processing, and modeling of lubrication system are realistic applications of squeezing flows [35] [36].

The boundary velocity, proportional to the shearing stress at the solid surface, is playing an important role in boundary value problems. For viscoelastic fluids, the slip condition is considerably important [37]. This feature has many applications in medical science, for example, polishing artificial heart valves [38]. There are several situations that include polymer fluids with high weight molecules, heavy suspensions, and lubrication problems flowing through multiple interfaces. Navier [39] initially proposed the general boundary condition which illustrates the fluid slip at the surface.

So far, few attempts have been made to study the transfer of heat and mass through a three-dimensional compression flow in a rotating channel, and therefore, the objective of the current work is to analyze the effect of thermal relaxation factor on the flow flux of time dependent Maxwell viscoelastic nanofluid that is squeezed in rotating parallel plates with porous stretched surface incorporating Cattaneo-Christov heat flux model.

\section{Mathematical Model}

The governing model equations consisting of conservation of mass, momentum, energy and concentration are given by

$$
\begin{gathered}
\frac{\partial \rho}{\partial t}+\nabla \cdot(\rho \boldsymbol{V})=0 \\
\rho \frac{\mathrm{d} \boldsymbol{V}}{\mathrm{d} t}=\nabla \cdot \tau \\
\rho c_{p} \frac{\mathrm{d} \boldsymbol{T}}{\mathrm{d} t}=-\nabla \cdot \boldsymbol{q}-p \nabla \cdot \boldsymbol{V}-c_{p} \boldsymbol{J}_{s} \cdot \nabla \boldsymbol{T}+\boldsymbol{\Phi}+\boldsymbol{S}_{Q} \\
\frac{\mathrm{d} \boldsymbol{C}}{\mathrm{d} t}=-\frac{1}{\rho} \nabla \cdot \boldsymbol{J}_{s}
\end{gathered}
$$

here, $\boldsymbol{V}=(u, v, w)$ is the three-dimensional velocity of the viscous fluid, $\tau$ is 
the Cauchy stress tensor, $\boldsymbol{T}$ is the temperature of the fluid, $\boldsymbol{q}$ is the heat flux, $\Phi$ is the viscous dissipation term that describes the conversion of mechanical energy to heat. Also, $\boldsymbol{S}_{Q}$ represents the heat sources, $\boldsymbol{J}_{s}$ is the sum of Brownian and thermophoresis diffusions, $\rho$ and $c_{p}$ are the density and specific heat respectively.

The elastico-viscous behavior of fluid will be realized if elastic stress is applied to the fluid, and the resulting strain will be time dependent characterized by relaxation time. The constitutive equation considering time dependent stress relaxation is [34]

$$
\tau=-p I+\boldsymbol{S}
$$

The extra stress tensor $\boldsymbol{S}$ satisfies the upper convected Maxwell model given by

$$
\boldsymbol{S}+\lambda_{S}\left(\frac{\mathrm{d} \boldsymbol{S}}{\mathrm{d} t}+\boldsymbol{V} \cdot \nabla \boldsymbol{S}-\boldsymbol{L} \boldsymbol{S}-\boldsymbol{S}^{\mathrm{tr}}\right)=\mu\left(\boldsymbol{L}+\boldsymbol{L}^{\mathrm{tr}}\right)
$$

here, $\boldsymbol{L}^{\mathrm{tr}}=\nabla \boldsymbol{V} \quad$ (i.e., $\left.\quad L_{i j}=\partial u_{i} / \partial x_{j}\right)$ is the velocity gradient and the superscript tr indicates a transpose, $\mu$ is the viscosity, $\lambda_{S}>0$ is the stress relaxation time where $\lambda_{S}=0$ describes the Newtonian fluids.

Cattaneo-Christov model is proposed by adding thermal relaxation time in Fourier's Law, also called the modified Fourier heat conduction law, presented by [7]

$$
\boldsymbol{q}+\lambda_{T}\left(\frac{\partial \boldsymbol{q}}{\partial t}+\boldsymbol{V} \cdot \nabla \boldsymbol{q}-\boldsymbol{q} \cdot \nabla \boldsymbol{V}+(\nabla \cdot \boldsymbol{V}) \boldsymbol{q}\right)=-\kappa \nabla \boldsymbol{T}
$$

here, $\kappa$ is the thermal conductivity and $\lambda_{T}$ is the thermal relaxation time parameter for the heat flux where $\lambda_{T}=0$ simplifies the expression (7) to classical Fourier's law.

Buongiorno [32] disclosed the combination of Brownian and thermophoresis diffusions given by

$$
\boldsymbol{J}_{s}=-\rho D_{B} \nabla \boldsymbol{C}-\rho D_{T} \frac{\nabla \boldsymbol{T}}{T_{a}}
$$

here, $D_{B}$ is the Brownian diffusion coefficient, $D_{T}$ is the thermal diffusion coefficient and $T_{a}$ is the reference temperature.

To demonstrate the physical model of present analysis, it is considered that the flow is laminar, unsteady and three dimensional. An incompressible $(\nabla \cdot V=\mathbf{0})$, electrically conducting elastico-viscous Maxwell nanofluid is being squeezed between two infinite parallel plates rotating about $y$-axis. To explain the physical configuration, the Cartesian coordinate system is introduced such a way that $x$-axis is measured along the plate surface and $y$-axis is perpendicular to the plates, shown in Figure 1. There is a vital consideration that fluid properties will not deviate of in the $z$-direction i.e., $\frac{\partial}{\partial z}=0$. The gap width between those plates in the minimal separation region is taken as time dependent given by $h(t)=l \sqrt{1-\alpha t}$, where $l$ is the steady gap width and $\alpha$ is a constant having 


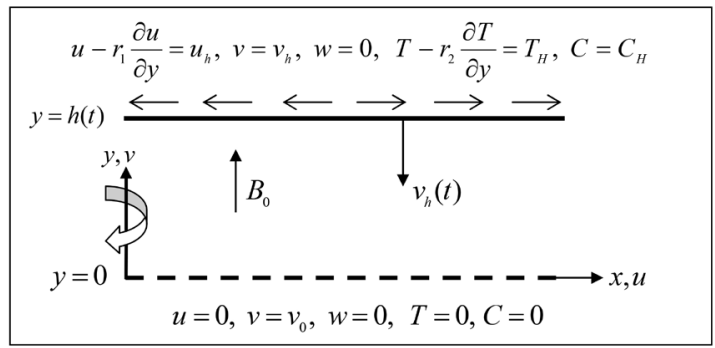

Figure 1. Physical model.

dimension time $_{-1}$. For $\alpha>0$ the two plates are squeezed until they touch $t=1 / \alpha$ and for $\alpha<0$ the two plates are always separated. The upper plate placed at $y=h(t)$ is squeezing towards the lower plate with a vertical velocity $v_{h}=\frac{\mathrm{d} h(t)}{\mathrm{d} t}$. This plate is stretched with a velocity $u_{h}=\alpha_{h} x /(1-\alpha t)$ in the positive $x$-direction with velocity slip parameter $r_{1}$ and thermal slip parameter $r_{2}$. The lower plate is fixed at $y=0$ assumed to be porous in which the fluid flows with suction velocity $v_{0}=-V_{0} /(1-\alpha t)$. A uniform magnetic field of density $B_{0}$ is applied to along $y$-direction and the external electric field is assumed zero.

The boundary conditions of the present physical models are

$$
\left.\begin{array}{l}
u-r_{1} \frac{\partial u}{\partial y}=u_{h}, v=v_{h}, w=0, T-r_{2} \frac{\partial T}{\partial y}=T_{H}, C=C_{H} \text { at } y=h(t) \\
u=0, v=v_{0}, w=0, T=0, C=0, \text { at } y=0
\end{array}\right\}
$$

Now in order to find the approximate solutions of the model it is essential to make the model equations dimensionless using the following non-dimensional variables [35] [36]:

$$
\begin{aligned}
& \eta=\frac{y}{l(1-\alpha t)^{\frac{1}{2}}}, u=\frac{\alpha x}{2(1-\alpha t)} f^{\prime}, v=\frac{-\alpha l}{2(1-\alpha t)^{\frac{1}{2}}} f, \\
& w=\frac{\alpha x}{2(1-\alpha t)} g, T=T_{H} \theta, C=C_{H} F
\end{aligned}
$$

Using the above transformations, the Equation (1) is satisfied and the Equations (2)-(4) are reduced to

$$
\begin{aligned}
& \left(1-S q \beta_{S}\left(\eta^{2}-2 \eta f+f^{2}\right)\right) f^{i v} \\
& -S q \beta_{S}\left(9 \eta f^{\prime \prime \prime}+2 \eta f^{\prime \prime 2}-2 f f^{\prime \prime 2}-2 f^{\prime 2} f^{\prime \prime}-8 f f^{\prime \prime \prime}+4 f^{\prime} f^{\prime \prime}+15 f^{\prime \prime}\right) \\
& -M\left(f^{\prime \prime}+\beta_{S}\left(\eta f^{\prime \prime \prime}+3 f^{\prime \prime}-f f^{\prime \prime \prime}-f^{\prime} f^{\prime \prime}\right)\right) \\
& -S q\left(\eta f^{\prime \prime \prime}+3 f^{\prime \prime}+f^{\prime} f^{\prime \prime}-f f^{\prime \prime \prime}+2 \omega g^{\prime}\right)=0 \\
& \quad\left(1-S q \beta_{S}\left(\eta^{2}-2 \eta f+f^{2}\right)\right) g^{\prime \prime} \\
& \quad-S q \beta_{S}\left(7 \eta g^{\prime}+2 \eta f^{\prime} g^{\prime}+4 f^{\prime} g-6 f g^{\prime}-2 f f^{\prime} g^{\prime}+8 g\right) \\
& \quad-S q\left(\eta g^{\prime}+2 g+f^{\prime} g-f g^{\prime}-2 \omega f^{\prime}\right) \\
& \quad-M\left(g+\beta_{S}\left(\eta g^{\prime}+2 g-f g^{\prime}\right)\right)=0
\end{aligned}
$$




$$
\begin{aligned}
& \left(1-\operatorname{Pr} S q \beta_{T}\left(\eta^{2}-2 \eta f+f^{2}\right)\right) \theta^{\prime \prime}-\operatorname{SqPr}\left(\eta \theta^{\prime}-f \theta^{\prime}\right) \\
& -\operatorname{SqPr} \beta_{T}\left(3 \eta \theta^{\prime}-\eta f^{\prime} \theta^{\prime}-3 f \theta^{\prime}+f f^{\prime} \theta^{\prime}\right) \\
& +\operatorname{Pr}\left(N_{B} \theta^{\prime} F^{\prime}+N_{T} \theta^{\prime 2}\right)+E c \operatorname{Pr}\left(4 \delta^{2} f^{\prime 2}+f^{\prime \prime 2}+g^{\prime 2}+\delta^{2} g^{2}\right)=0 \\
& F^{\prime \prime}-\operatorname{SqLe}\left(\eta F^{\prime}-f F^{\prime}\right)+\frac{N t}{N b} \theta^{\prime \prime}=0
\end{aligned}
$$

The dimensionless boundary conditions are

$$
\left.\begin{array}{l}
f=1, f^{\prime}=\gamma_{u}+\varepsilon_{u} f^{\prime \prime}, g=0, \theta=1+\varepsilon_{u} \theta^{\prime}, F=1, \text { at } \eta=1 \\
f=f_{w}, f^{\prime}=0, g=0, \theta=0, F=0 \text { at } \eta=0
\end{array}\right\}
$$

here, $\omega=\frac{2(1-\alpha t)}{\alpha} \Omega$ is the rotation parameter; $\beta_{S}=\frac{\lambda_{S} \alpha}{2(1-\alpha t)}$ is the Maxwell parameter; $S q=\frac{\alpha l^{2}}{2 v}$ is the squeeze number; $M=\frac{\sigma B_{0}^{2} h^{2}}{\mu}$ is the Magnetic field parameter; $\operatorname{Pr}=\frac{v \rho C p}{\kappa_{f}}$ is the Prandtl number; $E c=\frac{\rho u_{a}^{2}}{\rho C p T_{H}}$ is the Eckert number; $\gamma_{u}=\frac{\alpha_{h}}{\alpha}$ is the stretching parameter; $\varepsilon_{u}=\frac{r_{1}}{h}$ is the velocity slip parameter; $\varepsilon_{T}=\frac{r_{2}}{h}$ is the thermal slip parameter; $\delta=\frac{h}{x}$ is the characteristic length ratio; $f w=\frac{2 V_{0}}{\alpha h}$ is the suction parameter.

Finally, the physical attentions in the existing study are the skin friction coefficient $C_{f}$, the local Nusselt number $N u$ and the Sherwood number $(S h)$ defined as

$$
C_{f} \propto f^{\prime \prime}, N u \propto-\theta^{\prime} \text { and } S h \propto-F^{\prime}
$$

\section{Numerical Methods}

Equations (11)-(14) combined with the boundary conditions (15) are solved numerically using finite difference code developed by a MATLAB boundary value problem solver, known as bvp4c. The analysis is made for various values of the pertinent parameters such as Brownian motion parameter $N_{B}$, squeezing parameter $S q$, Maxwell parameter $\beta_{S}$, thermal relaxation parameter $\beta_{T}$, rotation parameter $\omega$, stretching parameter $\gamma_{u}$, velocity slip parameter $\varepsilon_{u}$ and thermal slip parameter $\varepsilon_{T}$. The step size is taken as $\eta=0.01$ and the tolerance criteria are set to $10^{-6}$. On the basis of the present model, [0,1] is measured as the domain of a channel. First of all, comparison of the current model is arranged with [35], shown in Table 1 .

\section{Results Discussions}

Figure 2(a) and Figure 2(b) are devoted to the analysis of the impact of the Brownian motion parameter $N_{B}$ on the temperature and concentration profiles, respectively. These figures allow us to conclude that the temperature and 
concentration distributions are enhanced with $N_{B}$. The Brownian motion parameter illustrates a significant variation in temperature profiles, while compared to concentration profiles. These outcomes express a similar result remarkable with the work of Reddy et al. [40]. These figures also reveals that the temperature of the fluid is lifted and the concentration is reduced for thermal relaxation parameter $\beta_{T}$.

Figure 3(a), Figure 3(b) and Figure 4(a), Figure 4(b) express the behavior of squeezing parameter $S q$. When plates are coming closer the values of $S q$ are considered positive. Figure 3(a) shows that, with the increase of the values of $S q$ fluid velocity decreases. Clearly the flow velocity decreases in the channel when fluid is clutched inside. But the secondary velocity profiles increase with $S q$, shown in Figure $3(\mathrm{~b})$. Furthermore, there is no variation in velocity profiles due to the classical Fourier's heat flux model $\left(\beta_{T}=0\right)$ and the Cattaneo-Christov heat flux model ( $\beta_{T}=0.3$ ). Figure 4 (a) and Figure 4(b) demonstrate the influence of $S q$ parameter on the heat and solutal distributions respectively. From the above representations, it can be revealed that the deviation of the fluid properties for the classical Fourier and Cattaneo-Christov heat fluxes approaches for the higher value of squeezing parameter.

Table 1. Comparison for skin friction coefficient, local Nusselt number and local Sherwood numbers for different values of $S q$ when $\operatorname{Pr}=E c=\gamma_{u}=1.0, \delta=0.1$.

\begin{tabular}{ccccccc}
\hline Lightaqua & \multicolumn{3}{c}{ Lightaqua Mustafa et al. [35] } & \multicolumn{3}{c}{ Lightaqua Present work } \\
\hline Lightaqua Pr & $-f^{\prime}(1)$ & $-\theta^{\prime}(1)$ & $F^{\prime}$ & $-f^{\prime}(1)$ & $-\theta^{\prime}(1)$ & $F^{\prime}$ \\
\hline-1.0 & 3.026324 & 3.02632355855 & 3.026323 & 2.170091 & 3.319899 & 0.804559 \\
-0.5 & 5.98053 & 5.98053039715 & 5.98053 & 2.617404 & 3.129491 & 0.781402 \\
0.01 & 14.43941 & 14.4394132325 & 14.439411 & 3.007133 & 3.047091 & 0.761225 \\
0.5 & 1.513162 & 1.51316180648 & 1.513161 & 3.336448 & 3.026327 & 0.744224 \\
2.0 & 3.631588 & 3.63158826816 & 3.631587 & 4.167387 & 3.118553 & 0.701813 \\
\hline
\end{tabular}
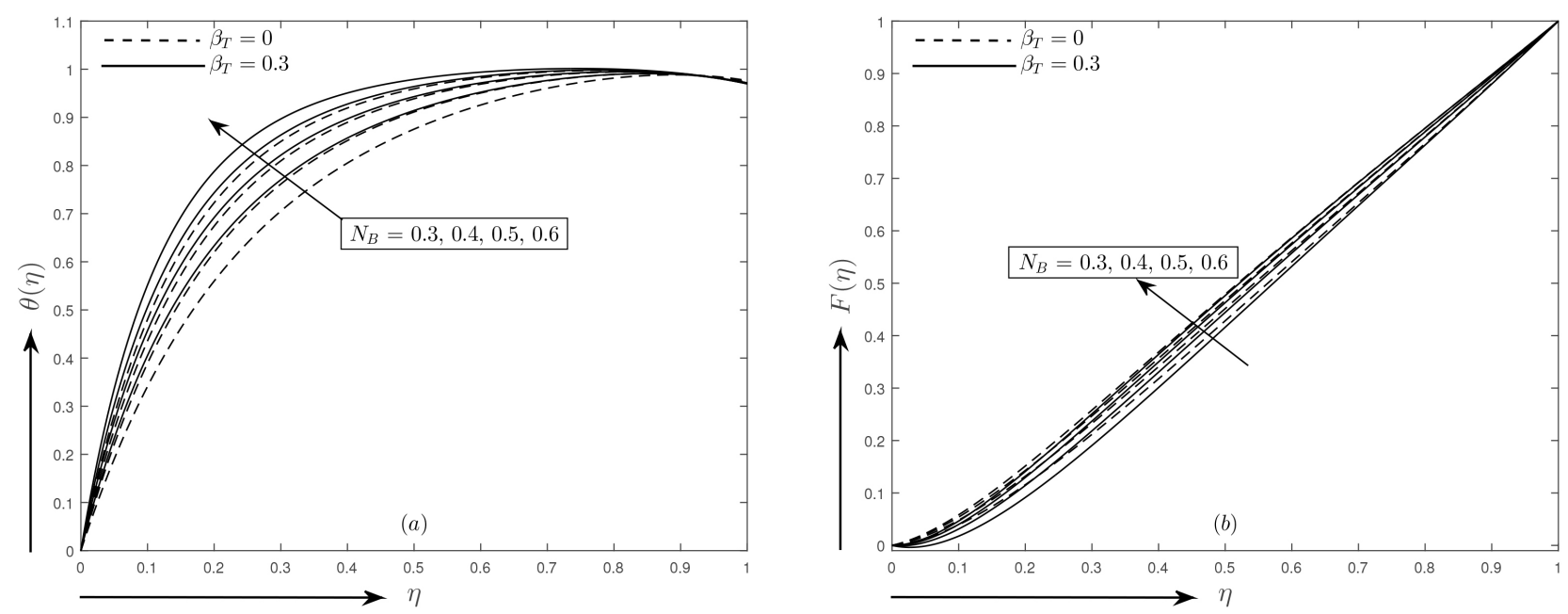

Figure 2. $N_{B}$ effect on the profiles of (a) temperature and (b) concentration. 

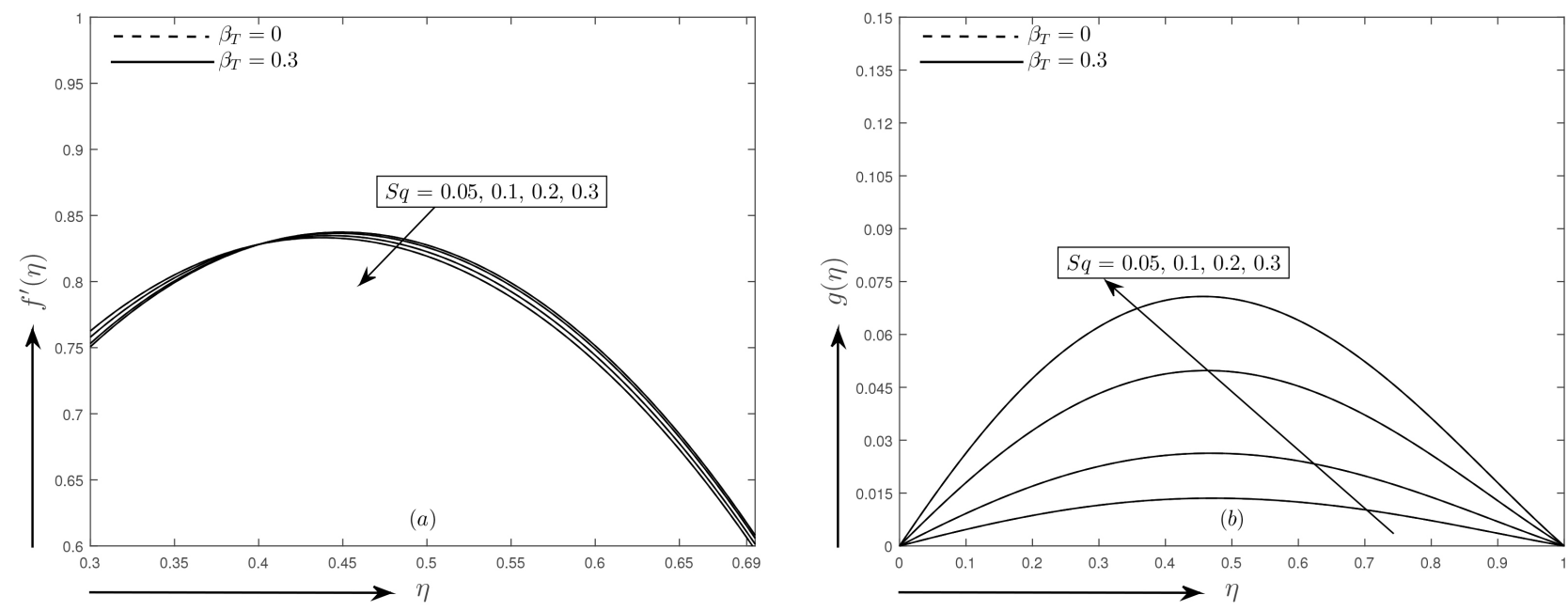

Figure 3. $S q$ effect on the profiles of (a) primary velocity and (b) transverse velocity.
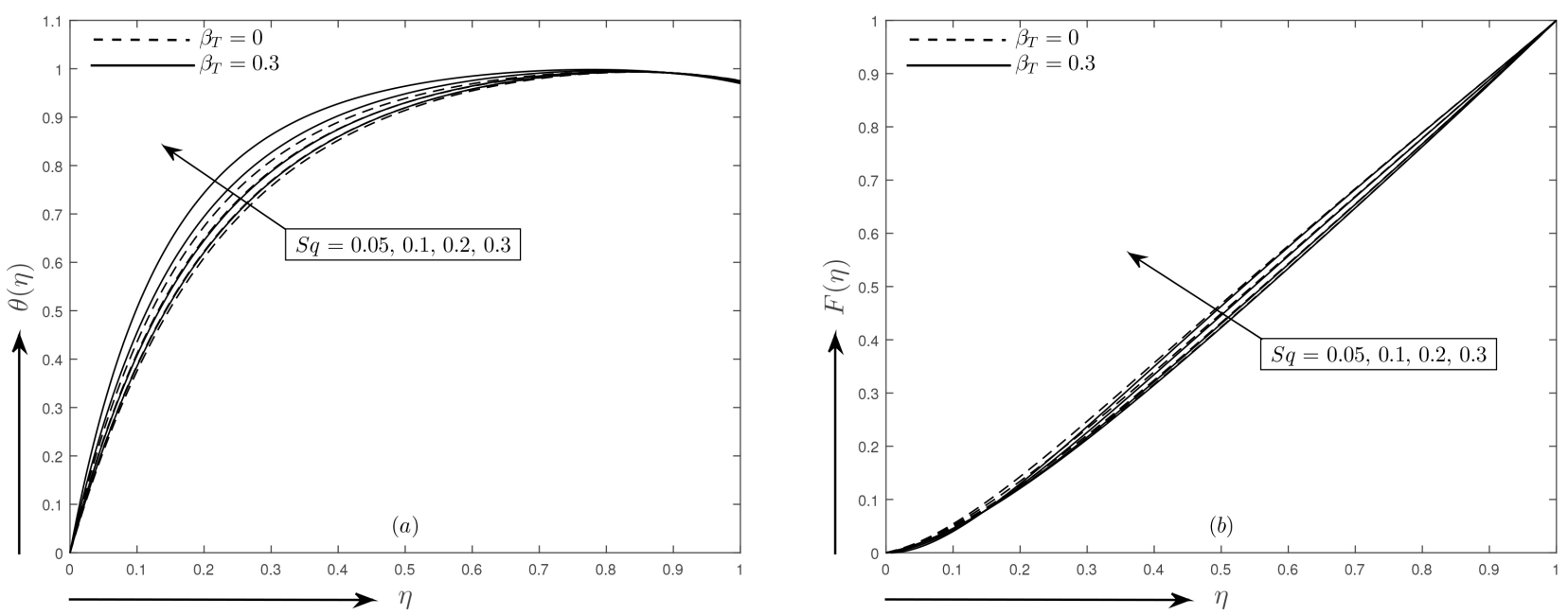

Figure 4. $S q$ effect on the profiles of (a) temeperature and (b) concentration.

When the elastic stress is applied to the non-Newtonian fluid, the time during which the fluid achieves its stability is the relaxation time, which is greater for highly viscous fluids. The Maxwell parameter $\beta_{S}$ deals with the fluid relaxation time to its characteristic time scale. Here $\beta_{S}=0$ gives the result for Newtonian viscous incompressible fluid. The fluid with a small Maxwell parameter exhibits liquid-like activities but large Maxwell parameter communicates with solid-like materials able to conduct and retain heat better. Therefore, it is observed physically that gradually increasing the Maxwell parameter can increase the fluid viscosity, which enhances resistance to flow and, as a result, the hydrodynamic boundary layer thickness reduces for Maxwell fluid, as shown in Figure 5(a). There is also declining effect of $\beta_{S}$ on the secondary velocity, displayed in Figure 5(b).

Figure 6(a) presents the significant effect of rotation parameter $\omega$ on secondary velocity. The rotation of the plates generates higher $z$-momentum. Fig- 
ure 6(b) and Figure 7(a), Figure 7(b) depict the stretching parameter $\gamma_{u}$ effects on $x$-momentum, $z$-momentum and energy distributions, respectively. From Figure 6(b), it is observed that the stretching velocity enhances strength to the fluid velocity to increase the $x$-momentum distribution with the increase of stretching effect $\gamma_{u}$ at the stretching upper surface. Figure 7(a) expresses that the $z$-momentum decreases with the higher values of stretching parameter $\gamma_{u}$. Figure 7 (b) exhibits the fact that The energy distribution is an increasing function of $\gamma_{u}$.

The result found from Figure 8(a) expresses the fact that the velocity increases with the mounting value of velocity slip parameter $\varepsilon_{u}$ but after the cross flow situated at $\eta=0.656$ the velocity reverses the flow tendency and decreases at the upper wall indicated in the Figure 8(a). In Figure 8(b), it is found that the secondary velocity increases with the positive values of slip parameter $\varepsilon_{u}$. A significant depiction, portrayed from the above observations, the fluid velocity is higher for the velocity slip parameter $\varepsilon_{u}$. The energy distribution rises with the velocity slip parameter $\varepsilon_{u}$, found in Figure 9(a). But Figure 9(b) shows that the temperature profile is a decreasing function of the thermal slip parameter $\varepsilon_{T}$.

Finally, from the point of view of physical interest, the skin friction coefficient is useful to estimate the total frictional drag exerted on the surface. The Nusselt Number is used to characterize the heat flux from a heated solid surface to a fluid. Additionally, Table 2 displays the skin friction $\left(C_{f}\right)$ for squeezing parameters and Maxwell parameter at the upper $(\eta=1)$ and lower $(\eta=0)$ plates for classical Fourier $\left(\beta_{T}=0\right)$ and Cattaneo-Christov $\left(\beta_{T}=0.3\right)$ heat fluxes. The effect of squeezing parameter $S q$ and Maxwell parameter $\beta_{T}$ on the local Nusselt number $N u$ and are arranged in Table 3 classical Fourier $\left(\beta_{T}=0\right)$ and Cattaneo-Christov ( $\beta_{T}=0.3$ ) heat fluxes considering $M=1 ; \omega=2 ; \delta=0.1 ; \operatorname{Pr}=6.838 ; E c=0.02 ; \gamma_{u}=0.1 ; \varepsilon_{u}=0.1$.
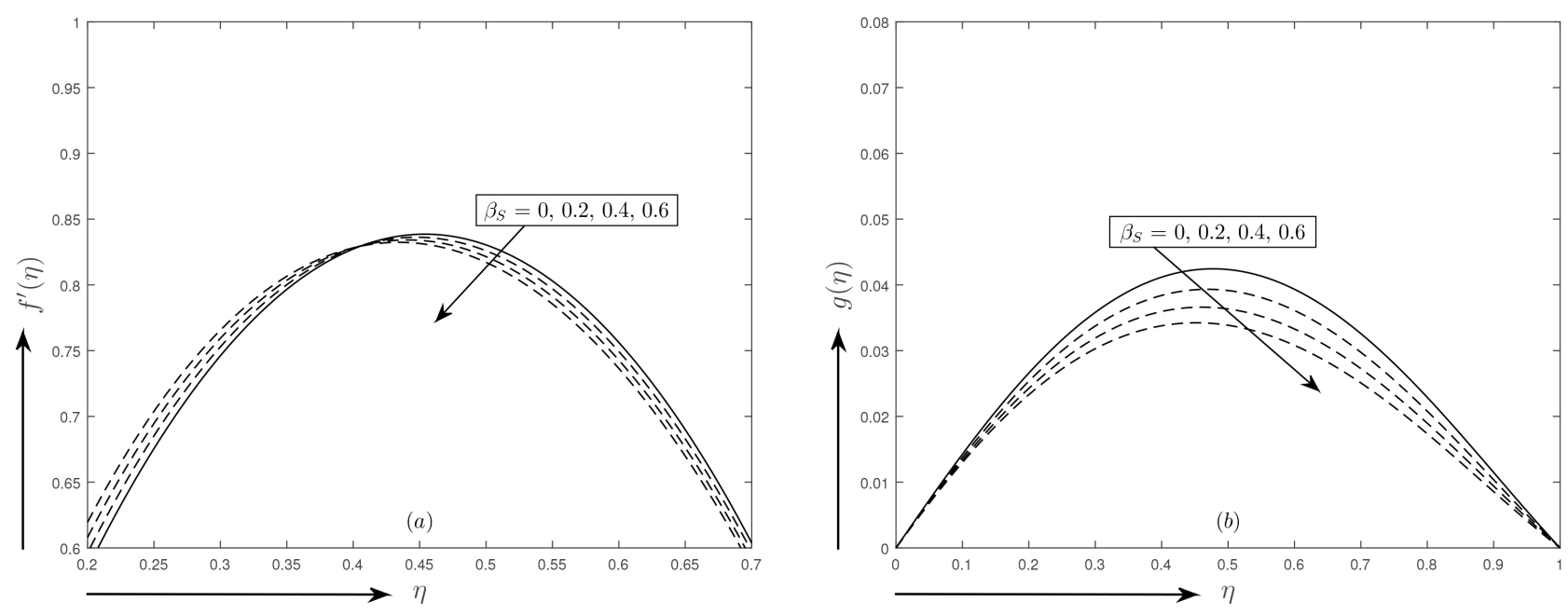

Figure 5. $\beta_{S}$ effect on the profiles of (a) primary velocity and (b) secondary velocity. 

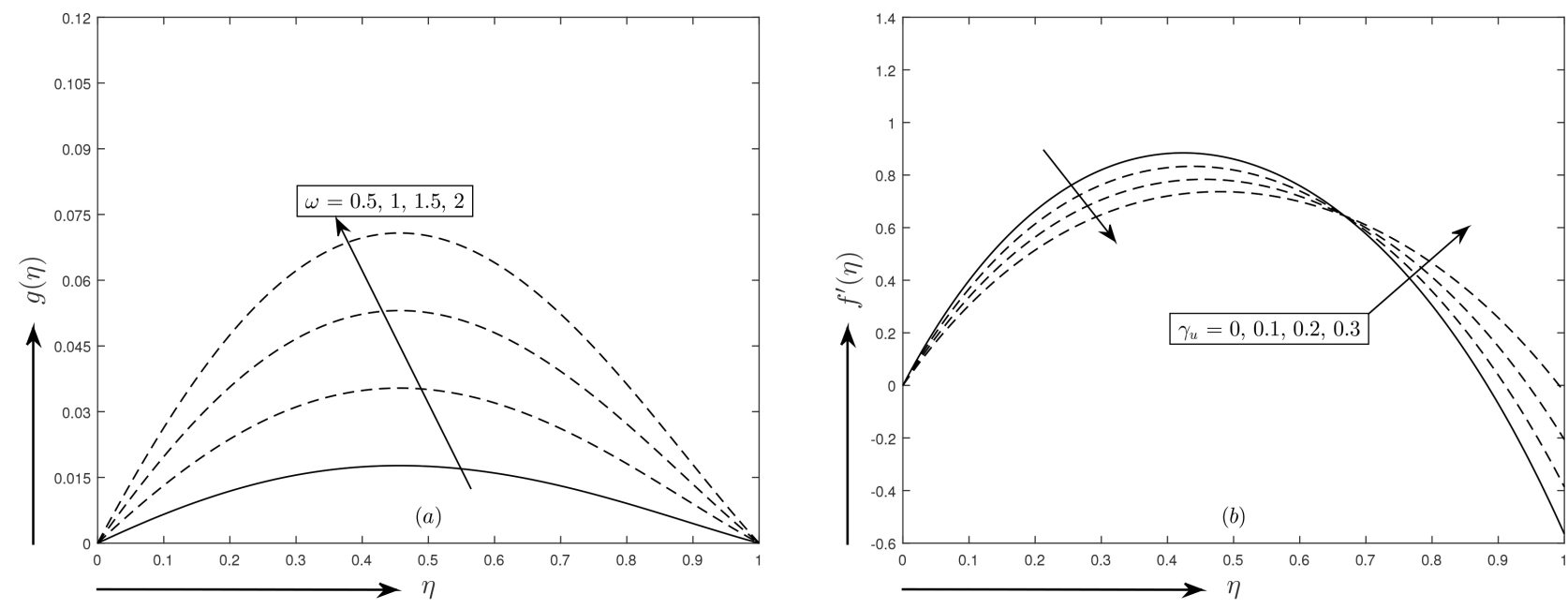

Figure 6. (a) $\varepsilon_{u}$ effect on transverse velocity profile and (b) $\gamma_{u}$ effect on primary velocity profile.
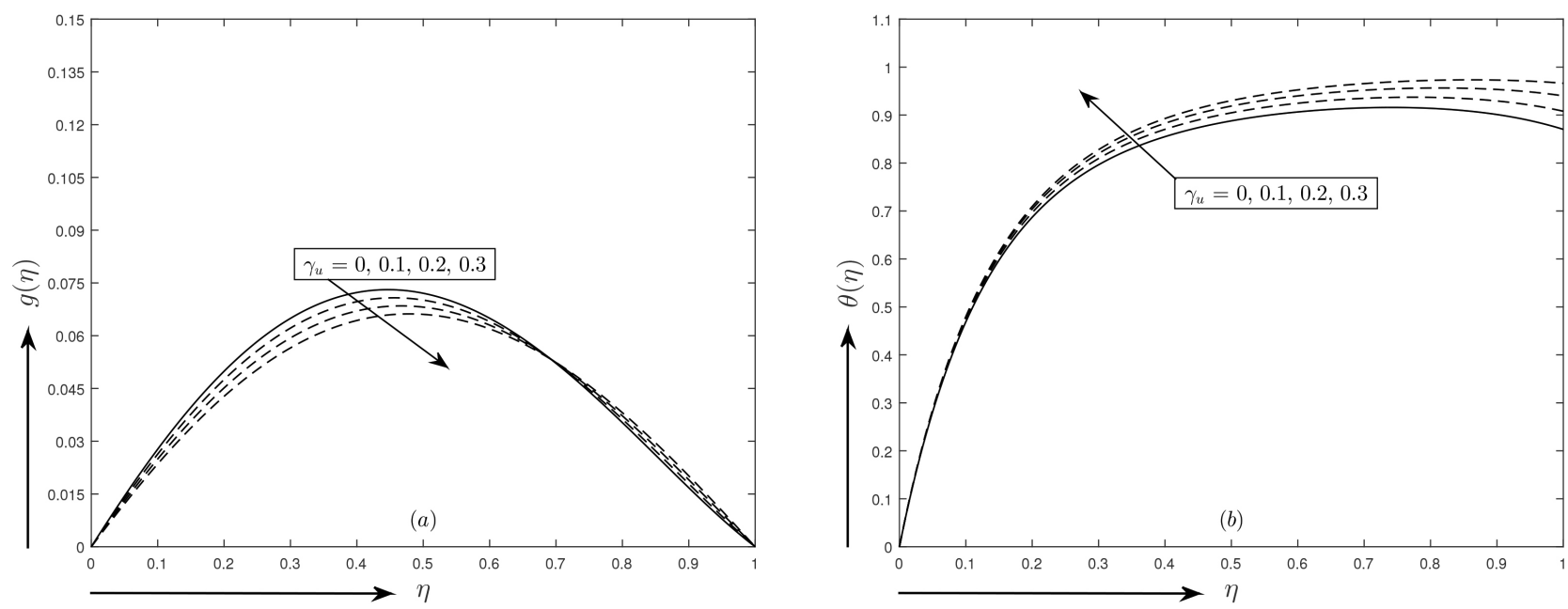

Figure 7. $\gamma_{u}$ effect on the profiles of (a) transverrse velocity and (b) temperature.
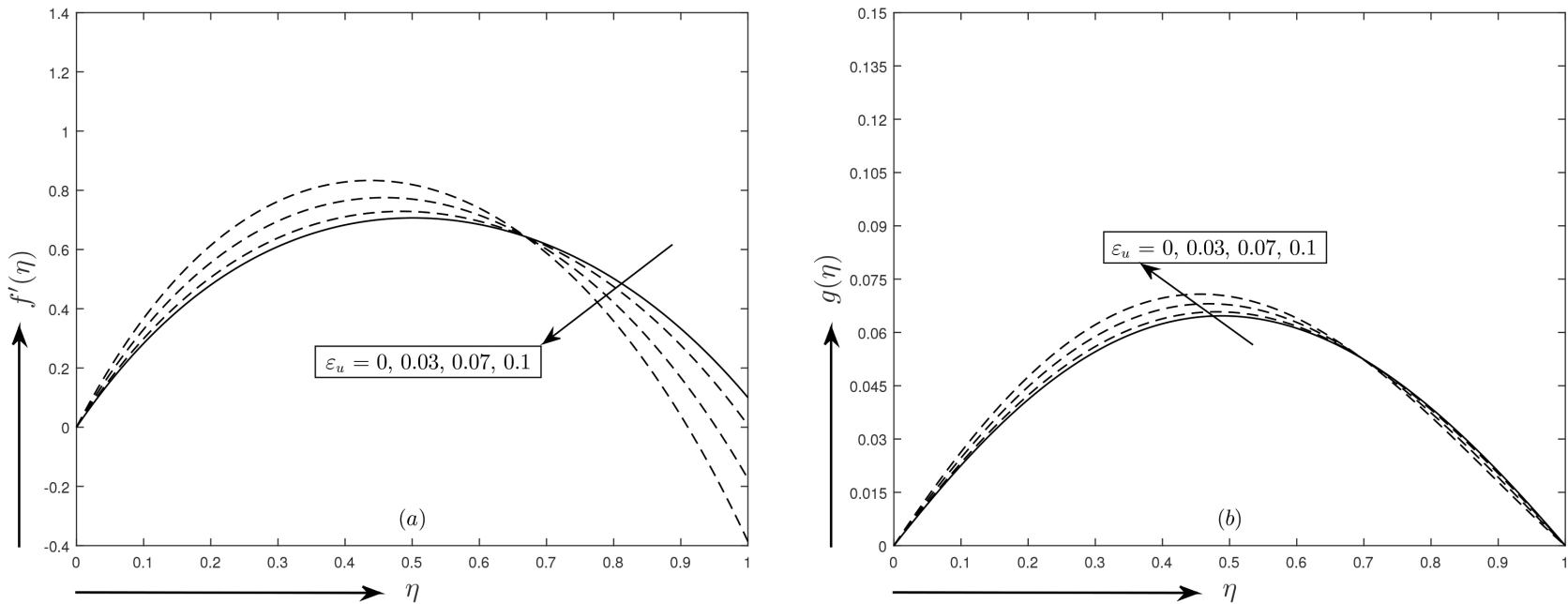

Figure 8. $\varepsilon_{u}$ effect on the profiles of (a) primary velocity and (b) secondary velocity. 

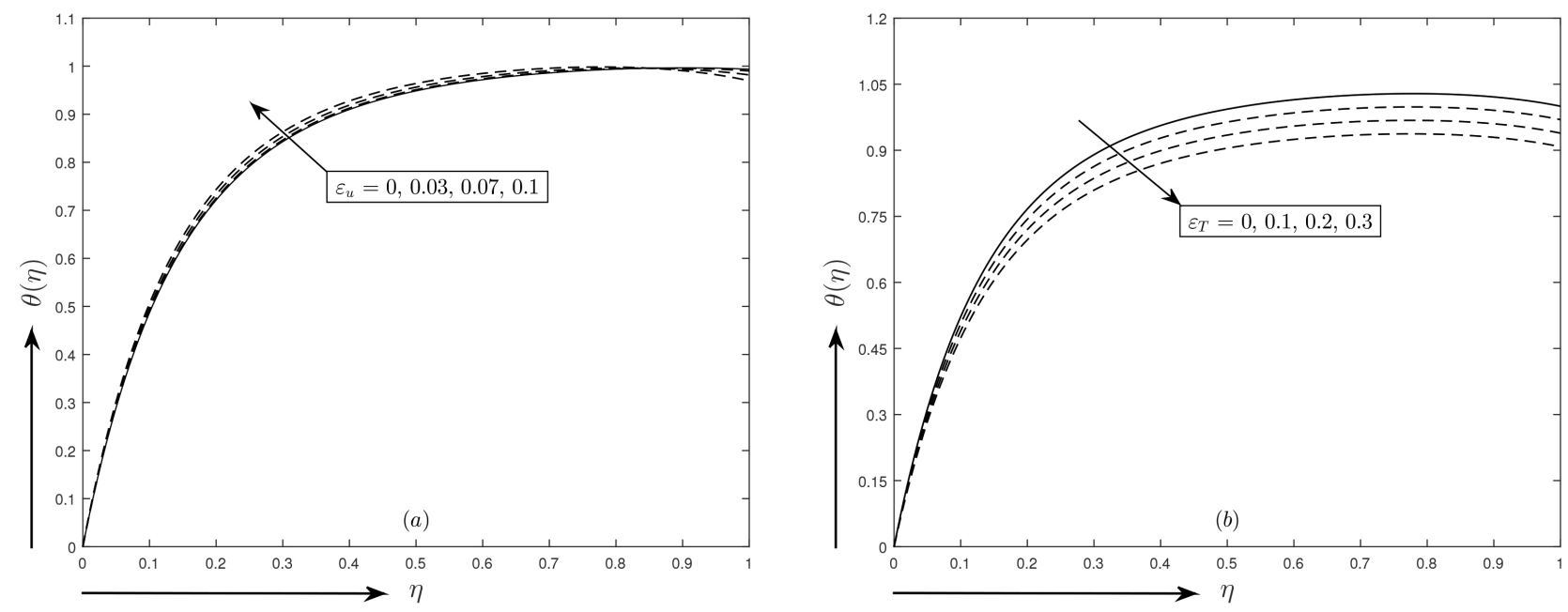

Figure 9. (a) $\varepsilon_{u}$ effect on the temperature profile and (b) $\varepsilon_{T}$ effect on the temperature profile.

Table 2. Skin friction $\left(C_{f}\right)$ for different values of $S q$ and $\beta_{S}$ at the upper $(\eta=1)$ and lower $(\eta=0)$ plates for classical Fourier $\left(\beta_{T}=0\right)$ and Cattaneo Christov $\left(\beta_{T}=0.3\right)$ heat fluxes.

\begin{tabular}{cccccc}
\hline & \multicolumn{2}{c}{$\beta_{T}=0$} & \multicolumn{2}{c}{$\beta_{T}=0.3$} \\
\hline$S q$ & $\beta_{S}$ & $C_{f_{n=1}}$ & $C_{f_{n=0}}$ & $C_{f_{n=1}}$ & $C_{f_{n=0}}$ \\
\hline 0.1 & 0.5 & -4.659543 & 4.056332 & -4.659543 & 4.056332 \\
0.2 & & -4.757606 & 4.214218 & -4.757606 & 4.214218 \\
0.3 & & -4.861431 & 4.377962 & -4.861431 & 4.377962 \\
& 0.6 & -4.921623 & 4.486911 & -4.921623 & 4.486911 \\
& 0.7 & -4.983358 & 4.598035 & -4.983358 & 4.598035 \\
\hline
\end{tabular}

Table 3. Nussult number $(\mathrm{Nu})$ for different values of $S q$ and $\beta_{S}$ at the upper $(\eta=1)$ and lower $(\eta=0)$ plates for classical Fourier $\left(\beta_{T}=0\right)$ and Cattaneo Christov $\left(\beta_{T}=0.3\right)$ heat fluxes.

\begin{tabular}{cccccc}
\hline & \multicolumn{2}{c}{$\beta_{T}=0$} & \multicolumn{2}{c}{$\beta_{T}=0.3$} \\
\hline$S q$ & $\beta_{S}$ & $N u_{\eta=1}$ & $N u_{\eta=0}$ & $N u_{\eta=1}$ & $N u_{\eta=0}$ \\
\hline 0.1 & 0.5 & 0.253098 & -4.942629 & 0.261092 & -5.325179 \\
0.2 & & 0.269455 & -5.387022 & 0.283571 & -6.271893 \\
0.3 & & 0.286328 & -5.861222 & 0.304701 & -7.396247 \\
& 0.6 & 0.290002 & -5.867768 & 0.308438 & -7.405840 \\
& 0.7 & 0.293886 & -5.874447 & 0.312386 & -7.415598 \\
\hline
\end{tabular}

\section{Conclusions}

The present paper is to study the effect of thermal relaxation factor on the flow of Maxwell nanofluid squeezing in the parallel rotating plates with porous stretched surface incorporating Cattaneo-Christov heat flux model. The major 
outcomes drawn from the study of the present model can be summarized as follows:

1) The thermal boundary layer thickness rises for the Brownian motion parameter, squeezing parameter, stretching parameter and velocity slip parameter.

2) The thermal boundary layer thickness decreases for the thermal slip parameter.

3) The hydrodynamic boundary layer thickness is reduced for the squeezing parameter, Maxwell parameter and stretching parameter.

4) The velocity distributions are higher for the velocity slip parameter.

5) The concentration is elevated for Brownian motion parameter and squeezing parameter.

In conclusion of the current study, it can be argued that the squeezing parameter and the stretching parameter that have the velocity control phenomena, can improve the heat transfer in the nanofluid. This study will provide a great opportunity to develop the cooling performance of mechanical system like automotive radiators and nuclear reactors.

\section{Acknowledgements}

We thank the Editor and the referee for their comments. Their support is greatly appreciated.

\section{Conflicts of Interest}

The authors declare no conflicts of interest regarding the publication of this paper.

\section{References}

[1] Fourier, J. (1822) Theorie analytique de la chaleur, par M. Fourier Chez Firmin Didot, père et fils.

[2] Cattaneo, C. (1948) Sulla conduzione del calore. Attidel Seminario Matematico e Fisicodella Università di Modena, 3, 83-101.

[3] Christov, C.I. (2009) On Frame Indifferent Formulation of the Maxwell-Cattaneo Model of Finite-Speed Heat Conduction. Mechanics Research Communications, 36, 481-486. https://doi.org/10.1016/j.mechrescom.2008.11.003

[4] Alamri, S.Z., Khan, A.A., Azeez, M. and Ellahi, R. (2019) Effects of Mass Transfer on MHD Second Grade Fluid towards Stretching Cylinder: A Novel Perspective of Cattaneo-Christov Heat Flux Model. Physics Letters A, 383, 276-281. https://doi.org/10.1016/j.physleta.2018.10.035

[5] Ciarletta, M. and Straughan, B. (2010) Uniqueness and Structural Stability for the Cattaneo-Christov Equations. Mechanics Research Communications, 37, 445-447. https://doi.org/10.1016/j.mechrescom.2010.06.002

[6] Tibullo, V. and Zampoli, V. (2011) A Uniqueness Result for the Cattaneo-Christov Heat Conduction Model Applied to Incompressible Fluids. Mechanics Research Communications, 38, 77-79. https://doi.org/10.1016/j.mechrescom.2010.10.008

[7] Han, S., Zheng, L., Li, C. and Zhang, X. (2014) Coupled Flow and Heat Transfer in Viscoelastic Fluid with Cattaneo-Christov Heat Flux Model. Applied Mathematics 
Letters, 38, 87-93. https://doi.org/10.1016/j.aml.2014.07.013

[8] Layek, G.C. and Pati, N.C. (2017) Bifurcations and Chaos in Convection Taking Non-Fourier Heat-Flux. Physics Letters A, 381, 3568-3575.

https://doi.org/10.1016/j.physleta.2017.09.020

[9] Upadhya, S., Mamatha and Raju, C.S.K. (2018) Cattaneo-Christov Heat Flux Model for Magnetohydrodynamic Flow in a Suspension of Dust Particles towards a Stretching Sheet. Nonlinear Engineering, 7, 237-246. https://doi.org/10.1515/nleng-2017-0162

[10] Al Sulti, F. (2019) Impact of Cattaneo-Christov Heat Flux Model on Stagnation-Point Flow toward a Stretching Sheet with Slip Effects. Journal of Heat Transfer, 141, Article ID: 022003. https://doi.org/10.1115/1.4041959

[11] Pinkus, O. and Sternlicht, B. (1949) Theory of Hydrodynamic Lubrication. McGraw-Hill, New York.

[12] Oldroyd, J.G. (1950) On the Formulation of Rheological Equations of State. Proceedings of the Royal Society of London. Series A. Mathematical and Physical Sciences, 200, 523-541. https://doi.org/10.1098/rspa.1950.0035

[13] Cameron, A. and Mc Ettles, C.M. (1981) Basic Lubrication Theory. Ellis Horwood, New York.

[14] Brust, M., Schaefer, C., Doerr, R., Pan, L., Garcia, M., Arratia, P.E. and Wagner, C. (2013) Rheology of Human Blood Plasma: Viscoelastic versus Newtonian Behavior. Physical Review Letters, 110, Article ID: 078305. https://doi.org/10.1103/PhysRevLett.110.078305

[15] Li, X.K., Luo, Y., Qi, Y. and Zhang, R. (2011) On Non-Newtonian Lubrication with the Upper Convected Maxwell Model. Applied Mathematical Modelling, 35, 2309-2323. https://doi.org/10.1016/j.apm.2010.11.003

[16] Sochi, T. (2010) Flow of Non-Newtonian Fluids in Porous Media. Journal of Polymer Science Part B: Polymer Physics, 48, 2437-2767.

https://doi.org/10.1002/polb.22144

[17] Fox, V.G., Erickson, L.E. and Fan, L.T. (1969) The Laminar Boundary Layer on a Moving Continuous Flat Sheet Immersed in a Non-Newtonian Fluid. AIChE Journal, 15, 327-333. https://doi.org/10.1002/aic.690150307

[18] Rajagopal, K.R., Na, T.Y. and Gupta, A.S. (1984) Flow of a Viscoelastic Fluid over a Stretching Sheet. Rheologica Acta, 23, 213-215. https://doi.org/10.1007/BF01332078

[19] Andersson, H.I., Bech, K.H. and Dandapat, B.S. (1992) Magnetohydrodynamic Flow of a Power-Law Fluid over a Stretching Sheet. International Journal of Non-Linear Mechanics, 27, 929-936. https://doi.org/10.1016/0020-7462(92)90045-9

[20] Sadeghy, K. and Sharifi, M. (2004) Local Similarity Solution for the Flow of a Second-Grade Viscoelastic Fluid above a Moving Plate. International Journal of Non-Linear Mechanics, 39, 1265-1273.

https://doi.org/10.1016/j.ijnonlinmec.2003.08.005

[21] Bhattacharyya, K., Hayat, T. and Gorla, R.S.R. (2013) Heat Transfer in the Boundary Layer Flow of Maxwell Fluid over a Permeable Shrinking Sheet. Thermal Energy and Power Engineering, 2, 72-78.

[22] Hayat, T., Abbas, Z. and Sajid, M. (2006) Series Solution for the Upper-Convected Maxwell Fluid over a Porous Stretching Plate. Physics Letters A, 358, 396-403. https://doi.org/10.1016/j.physleta.2006.04.117

[23] Alarifi, I.M., Abokhalil, A.G., Osman, M., Lund, L.A., BenAyed, M., Belmabrouk, H. and Tlili, I. (2019) MHD Flow and Heat Transfer over Vertical Stretching Sheet 
with Heat Sink or Source Effect. Symmetry, 11, 297. https://doi.org/10.3390/sym11030297

[24] Fetecau, C. and Fetecau, C. (2003) A New Exact Solution for the Flow of a Maxwell Fluid past an Infinite Plate. International Journal of Non-Linear Mechanics, 38, 423-427. https://doi.org/10.1016/S0020-7462(01)00062-2

[25] Harris, J. (1966) Some Engineering Aspects of Non-Newtonian Flow. Nature, 211, 579-581. https://doi.org/10.1038/211579a0

[26] Mustafa, M., Khan, J.A., Hayat, T. and Alsaedi, A. (2015) Simulations for Maxwell Fluid Flow past a Convectively Heated Exponentially Stretching Sheet with Nanoparticles. AIP Advances, 5, Article ID: 037133. https://doi.org/10.1063/1.4916364

[27] Halim, N.A. and Noor, N.F.M. (2015) Analytical Solution for Maxwell Nanofluid Boundary Layer Flow over a Stretching Surface. AIP Conference Proceedings, 1682, Article ID: 020006. https://doi.org/10.1063/1.4932415

[28] Choi, S. and Eastman, J.A. (1995) Enhancing Thermal Conductivity of Fluids with Nanoparticles. Argonne National Laboratory, Lemont.

[29] Wong, K.V. and De Leon, O. (2010) Applications of Nanofluids: Current and Future. Advances in Mechanical Engineering, 2, Article ID: 519659. https://doi.org/10.1155/2010/519659

[30] Uddin, M.J., Al Kalbani, K.S., Rahman, M.M., Alam, M.S., Al-Salti, N. and Eltayeb, I. (2016) Fundamentals of Nanofluids: Evolution, Applications and New Theory. International Journal of Biomathematics and Systems Biology, 2, 1-32.

[31] Kuznetsov, A.V. and Nield, D.A. (2010) Natural Convective Boundary-Layer Flow of a Nanofluid past a Vertical Plate. The International Journal of Thermal Sciences, 49, 243-247. https://doi.org/10.1016/j.ijthermalsci.2009.07.015

[32] Buongiorno, J. (2006) Convective Transport in Nanofluids. Journal of Heat Transfer, 128, 240-250. https://doi.org/10.1115/1.2150834

[33] Stefan, M.J. (1874) Versuch über die scheinbare Adhäsion. Sitzungsber Abt II. Ö sterr Akad Wiss Math-Naturwiss K1, 69, 713-721.

[34] Phan-Thien, N. and Tanner, R.I. (1983) Viscoelastic Squeeze Film Flows-Maxwell Fluids. Journal of Fluid Mechanics, 129, 265-281.

https://doi.org/10.1017/S0022112083000762

[35] Mustafa, M., Hayat, T. and Obaidat, S. (2012) On Heat and Mass Transfer in the Unsteady Squeezing Flow between Parallel Plates. Meccanica, 47, 1581-1589. https://doi.org/10.1007/s11012-012-9536-3

[36] Akmal, N., Sagheer, M. and Hussain, S. (2018) Numerical Study Focusing on the Entropy Analysis of MHD Squeezing Flow of a Nanofluid Model Using Cattaneo-Christov Theory. AIP Advances, 8, Article ID: 055201. https://doi.org/10.1063/1.5029959

[37] Le Roux, C. (1999) Existence and Uniqueness of the Flow of Second-Grade Fluids with Slip Boundary Conditions. Archive for Rational Mechanics and Analysis, 148, 309-356. https://doi.org/10.1007/s002050050164

[38] Jiang, Y., Qi, H., Xu, H. and Jiang, X. (2017) Transient Electroosmotic Slip Flow of Fractional Oldroyd-B Fluids. Microfluidics and Nanofluidics, 21, 7. https://doi.org/10.1007/s10404-016-1843-x

[39] Navier, C.L.M.H. (1823) Memoirs de l'Academie. Royale des Sciences de PInstitut de France, 1, 414-416.

[40] Reddy, J.V.R., Sugunamma, V. and Sandeep, N. (2018) Thermophoresis and Brow- 
nian Motion Effects on Unsteady MHD Nanofluid Flow over a Slendering Stretching Surface with Slip Effects. Alexandria Engineering Journal, 57, 2465-2473.

https://doi.org/10.1016/j.aej.2017.02.014 